\title{
Resumption of oral anticoagulation after spontaneous intracerebral hemorrhage
}

\author{
Jochen A. Sembill ${ }^{*}$, Joji B. Kuramatsu, Stefan Schwab and Hagen B. Huttner
}

\begin{abstract}
Background: Given an ageing population the incidence of both patients suffering from intracerebral hemorrhage $(\mathrm{ICH})$ and those requiring oral anticoagulation will increase. Up to now there are no results from randomized trials available whether or not, and when, ICH survivors should resume OAC. This review summarizes the most important observational studies, and initiated ongoing trials, to help guiding physicians in daily routine decision making.

Findings: Several large observational studies and meta-analyses verified that OAC resumption was associated with a significant reduction of thromboembolic complications and mortality without leading to increased rates of recurrent $\mathrm{ICH}$. OAC resumption seemed further associated with improved functional recovery and favorable long-term outcome. Given the general bleeding risk reduction in patients using Non-vitamin K antagonist oral anticoagulants (NOAC) compared to Vitamin-K-antagonist (VKA), NOAC use should also be preferred after ICH, although specific comparative studies are pending. Patients with lobar ICH need special attention as these patients showed increased ICH recurrence rates, why decision making should include extended diagnostic work-up evaluating cerebral microbleed burden, cortical subarachnoid hemorrhage and superficial siderosis. Further, patients with mechanical heart valves need specific consideration as restarting VKA may be unsafe until two weeks, whereas optimal balancing of hemorrhagic with thromboembolic complications may allow earlier re-initiation one week after ICH. In patients with atrial fibrillation, resumption generally should take place between 4 and 8 weeks after ICH depending on a patient's individual risk profile. Left atrial appendage occlusion (LAAO) might represent an alternative strategy in high-risk patients. Ongoing clinical trials will clarify whether OAC resumption versus $L A A O$ versus no antithrombotic therapy may represent the best possible secondary stroke prevention in $\mathrm{ICH}$ survivors with atrial fibrillation.
\end{abstract}

Conclusions: According to observational data OAC resumption after $\mathrm{ICH}$ seems beneficial and safe. Ongoing clinical trials will create evidence regarding treatment effects of pharmaceutical resumption and interventional alternatives. Yet, individual decision making weighing the patient's individual thromboembolic versus hemorrhagic risks remains essential.

Keywords: Intracerebral hemorrhage, Oral anticoagulation, Resumption, Stroke prevention, Secondary prophylaxis, Intracranial hemorrhage

\section{Background - intracerebral hemorrhage and oral anticoagulation}

Intracerebral hemorrhage ( $\mathrm{ICH})$ still lacks effective treatments to positively influence functional outcome of patients suffering this severe sub-type of stroke (10$15 \%)[16,39]$. The consequences are high mortality rates $(\sim 50 \%)$ and functional dependency in many survivors ( $2 / 3$ of patients) [16, 39]. Although global incidence rates are expected to increase, the improved control of cerebrovascular risk factors may have contributed to a

\footnotetext{
* Correspondence: Jochen.sembill@uk-erlangen.de

Department of Neurology, University Hospital Erlangen, Schwabachanlage 6, 91054 Erlangen, Germany
}

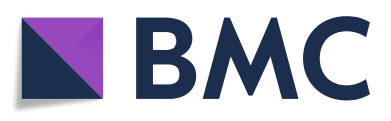

(c) The Author(s). 2019 Open Access This article is distributed under the terms of the Creative Commons Attribution 4.0 International License (http://creativecommons.org/licenses/by/4.0/), which permits unrestricted use, distribution, and reproduction in any medium, provided you give appropriate credit to the original author(s) and the source, provide a link to the Creative Commons license, and indicate if changes were made. The Creative Commons Public Domain Dedication waiver (http://creativecommons.org/publicdomain/zero/1.0/) applies to the data made available in this article, unless otherwise stated. tial future consequence hypertensive non-lobar ICH might occur less frequently than lobar ICH, the incidence of which is likely to rise with increasing rates of cerebral amyloid angiopathy (CAA) in an ageing society [5].

Likewise, rates of patients requiring oral anticoagulation $(\mathrm{OAC})$ for prevention of thromboembolism due to atrial fibrillation (AF) are rising. Both, use of OAC and prior $\mathrm{ICH}$, especially in lobar location, are known to increase the risk of recurrent intracerebral bleeding [6]. Both conditions occur coincidentally in almost one third of $\mathrm{ICH}$ patients - $15 \%$ of patients developed $\mathrm{ICH}$ already using $\mathrm{OAC}$ and another $15 \%$ have a de-novo diagnosis 
indicating future OAC prescription $[24,32,34,46]$. Hence, question remains whether physicians should (re-)start a formally contraindicated treatment in high-risk patients already having experienced $\mathrm{ICH}$ as the most feared adverse OAC drug reaction.

\section{Anticoagulation resumption - a no-win situation?}

The difficult decision whether or not to resume OAC after ICH is based on weighing patient's individual risk for ischemic complications due to thromboembolism versus hemorrhagic complications, above all recurrent $\mathrm{ICH}$. Due to low quality of evidence during time of preparation, the current 2014's \& 2015's guidelines American Heart Association/American Stroke Association, European Stroke Organization - do only provide little help, suggesting a multidisciplinary approach for individual decision making $[16,48]$. While the benefit of $\mathrm{OAC}$ for prevention of thromboembolic complications, caused by several indications like atrial fibrillation, artificial heart valves, deep vein thrombosis and pulmonary embolism, or coagulopathies, is generally accepted, OAC resumption after $\mathrm{ICH}$ is mainly an issue of safety, i.e. risk of recurrent $\mathrm{ICH}$ [25].

Recurrence risk in general is related to several modifiable and non-modifiable factors. Patient age and stroke history represent non-modifiable risk factors for both thromboembolic and hemorrhagic complications, reflected by their simultaneous integration into commonly used stratification models $\left(\mathrm{CHADS}_{2}\right.$-Score [0-6, from low to high stroke risk in $\mathrm{AF}$ patients; congestive heart failure $=1$, arterial hypertension $=1$, age $\geq 75=1$, diabetes mellitus $=1$, previous stroke or transient ischemic attack $=2$ ] [12] and HAS-BLED-Score [0-9, from low to high major bleeding risk on anticoagulation; uncontrolled arterial hypertension $=1$, renal disease $=1$, liver disease $=1$, history of stroke $=1$, prior major bleeding $=1$, unstable INR $=1$, age $>65=1$, use of drugs predisposing to bleeding $=1$, alcohol use =1]) [37]. The same holds true for (uncontrolled, > $140 \mathrm{mmHg}$ ) arterial hypertension representing a major - but importantly modifiable - risk factor especially for recurrence of $\mathrm{ICH}$, increasing hazard ratios [HR] to $3.5(95 \% \mathrm{CI}(1.7-7.5), p=.001)$ in lobar $\mathrm{ICH}$ and to 4.2 (95\% CI $(1.0-17.5), p<.05)$ in non-lobar ICH [1]. Distinguishing location of index ICH is of outmost importance because of the strong relation between lobar ICH and cerebral amyloid angiopathy [25]. Lobar location increases the risk of recurrent $\mathrm{ICH}$ shown by longitudinal data $(n=1145)$ documenting a duplication of the annual recurrence rate compared to non-lobar ICH (7.8\% versus 3.4\%) [1]. In concordance, a meta-analysis of 9 cohort studies including 1552 patients investigated the first ever ICH risk in relation to cerebral microbleed status in patients with ischemic stroke and atrial fibrillation using long-term OAC [9].
The annual ICH incidence rate rose from $0.3 \%$ in patients without microbleeds to $0.8 \%$ in patients with any microbleeds and to $2.5 \%$ in patients with more than 5 microbleeds [9]. Moreover, a vast number of additional factors - gender, diabetes mellitus, serum lipid levels, smoking, alcohol or drug abuse, and further medication subtly interacting with coagulation and platelet function - have been documented to be associated with ICH recurrence, complicating the decision if, when and how to resume OAC after ICH [25].

\section{Current evidence \\ Resuming oral anticoagulation after ICH}

After few and small-sized case series, the first larger retrospective observational analysis was published by Majeed et al. who investigated 234 patients with intracranial hemorrhage including 83 patients with ICH $(55 \%, n=83 / 234)$ documenting an overall increased risk for intracranial hemorrhage recurrence if OAC was resumed (11.5\% versus $17.8 \%)$ [28]. In contrast, data from a Canadian registry (including $89 \%$ ICH patients, $n=252 / 284$ ) reported annual recurrence rates less than $2.5 \%$ as well as a decreased mortality in patients resuming OAC [56]. Similar, a large Italian multicenter study documented an annual recurrence rate of $2.6 \%$ among 267 patients with intracranial hemorrhage resuming OAC (including 33\% ICH patients, $n=88 / 267$ ). All these studies remained inconclusive on whether or not risks for $\mathrm{ICH}$ recurrence were increased in a setting of OAC resumption [25].

From the year 2015 onwards there was growing evidence. The observational "geRman-widE mulTicenter Analysis of oRal Anticoagulation-associated intraCerebral hEmorrhage" (RETRACE) study included patients with $\mathrm{OAC}$-associated $\mathrm{ICH}$ and investigated thromboembolic and hemorrhagic complication rates according to OAC exposure during one year of follow-up [24]. Among 719 survivors with AF, resumption of OAC significantly reduced thromboembolic events (OAC: 9/ 172 [5.2\%] versus no-OAC: $82 / 547$ [15.0\%]; $p<0.001$ ) without leading to increased rates of re-bleeding (OAC: 14/172 [8.1\%] versus no-OAC: $36 / 547$ [6.6\%]; $p=0.48$ ) [24]. Furthermore, OAC resumption was associated with a decreased long-term mortality risk among patients included in a propensity-matched survival analysis (HR: 0.258 (95\% Confidence Interval $[\mathrm{CI}], 0.125-0.534 ; p<$ $0.001)$; i.e. 9 patients with $\mathrm{OAC}$ of 108 died (8.3\%) compared to 47 patients without OAC of 153 (30.7\%; $p<$ $0.001)$ [24].

The same year, a large Danish registry including 1752 patients reported data strongly supporting these results [32]. The authors found a significantly decreased adjusted HR [0.55, 95\% CI (0.39-0.78)] for all-cause mortality, stroke, and systemic embolism in patients on oral 
anticoagulant treatment in comparison with no treatment during 1-year follow-up [32]. The annual incidence rate of ischemic stroke and systemic embolism among patients using OAC was halved (5.3, 95\% CI (3.3-8.5) per 100 patient years) compared with patients without antithrombotic treatment (10.4) or on antiplatelet therapy (10.3). For recurrent $\mathrm{ICH}$, rates of 8.0 for OAC treated patients again did not significantly differ from 8.6 for patients with no antithrombotic treatment [adjusted HR, 0.91, 95\% CI (0.56-1.49)], and 5.3 for patients using antiplatelet therapy [adjusted HR, 0.60, 95\% CI (0.37-1.03)]. Another Danish population-based cohort study $(n=2978)$ confirmed these results, showing a significant lower risk of death [adjusted HR, 0.59, 95\% CI (0.43-0.82)] and thromboembolic events [adjusted HR, $0.5895 \%$ CI $(0.35-0.97)]$ in ICH patients with post-discharge use of $\mathrm{OAC}$, again without significantly increasing risks for major bleedings or recurrent $\mathrm{ICH}$ [adjusted HR $0.65,95 \%$ CI $(0.41-1.029$ ] [34]. These results favoring resumption of $\mathrm{OAC}$ were further reproduced by several subsequent observational and registry studies [35, 36, 53].

To this day, the largest registry study was conducted in Taiwan and included 12,917 patients with intracranial hemorrhage from 1996 to 2011, reporting divergent results [4]. Chao and colleagues documented an increased risk for hemorrhage recurrence for both patients resuming OAC [HR 1.58, 95\% CI (1.27-1.98)] as well as patients taking antiplatelet therapy [HR 1.36, 95\% CI (1.19-1.57)] after propensity score matched analyses [4]. According to the authors only patients with a major thromboembolic risk $\left(\mathrm{CHA}_{2} \mathrm{DS}_{2}\right.$-VASc-Score $\left.\geq 6\right)$ would have a net-benefit resuming warfarin, shown by comparison of the number needed to treat of 27 (for preventing one ischemic stroke) versus the number needed to harm of 91 (for producing one $\mathrm{ICH}$ ) [4]. However, these results might also be influenced by in general increased ICH risk in Asian patients [51].

Several meta-analyses of reported data have been conducted until now, all of them showing a significant reduction of thromboembolic complications without leading to increased risk of ICH recurrence [3, 22, 30, 57]. Furthermore, antiplatelet agents - sometimes considered as a safer alternative approach - were not beneficial neither for thromboembolism prophylaxis nor prevention of $\mathrm{ICH}$ recurrence [22]. One recent meta-analysis of individual patient data $(n=1012)$ did also address the association of OAC resumption with functional outcome, documenting that $\mathrm{OAC}$ resumption increases chances for a favorable outcome after 12 months (modified Rankin Scale 0-3) by 4-fold in both non-lobar and lobar ICH patients [2]. Even in the absence of recurrent clinically apparent stroke, patients resuming $\mathrm{OAC}$ seem to benefit with respect to better functional recovery, hypothetically due to prevention from micro- embolism cumulating to significant central nervous system damage influencing post-ICH recovery associated with cardioembolic stroke risk [29].

Of note, all of these observational studies harbor important limitations due to confounding by indication and selection bias [25]. Physicians individually weigh patient's risk for ischemic versus hemorrhagic complications which results in selected patients with favorable risk-benefit-profiles restarting OAC. This might also be reflected by their younger age, less severe $\mathrm{ICH}$, and better functional outcome in observational studies [24, 46]. In general, withholding therapy in severely affected patients is frequent in ICH care possibly further affecting post-discharge drug prescription $[44,46]$. As statistical adjustment is to a large extent possible for quantifiable parameters, additional unmeasured variables likely introduce residual bias influencing the reported associations $[22,46]$. Further, many investigations included heterogeneous patient cohorts combining different intracranial pathologies - mostly $\mathrm{ICH}$, but also patients with subarachnoid hemorrhage, epidural or subdural hematomas as well as OAC indications - atrial fibrillation, mechanical heart valves, deep vein thrombosis - each strongly influencing patients individual risk for recurrent hemorrhage or thromboembolism [25].

\section{Mode of resumption}

Although data from observational studies in the vast majority solely cover resumption of OAC using vitamin- $\mathrm{K}$ antagonists (VKA), it seems apparent that Non-vitamin K antagonist oral anticoagulants (NOAC) should be preferred [25]. Compared to VKA large randomized trials have demonstrated a halved $\mathrm{ICH}$ incidence in patients using NOAC $[10,20]$. Although the mechanism behind risk reduction is not completely understood, it seems that the more selective mode of action, i.e. targeting only one clotting factor, together with limited crossing of the blood-brain barrier (dabigatran) or effluxing out of the brain by p-glycoprotein efflux pumps (rivaroxaban and apixaban) lead to a more beneficial safety profile [55]. Recently, a Bayesian network meta-analysis including 17 randomized controlled trials enrolling 116,618 patients evidenced that all NOACs were safer than warfarin for risk of ICH [55]. Relative ranking probability based on surface under the cumulative ranking curve (SUCRA) suggested the safest profile among NOACs for dabigatran (SUCRA, 0.86) followed by edoxaban (SUCRA, 0.81), apixaban (SUCRA, 0.61), and rivaroxaban (SUCRA, 0.32). VKA was ranked as the least safe drug among all anticoagulants (SUCRA, 0.06). In general, NOACs were associated with a significant $54 \%$ relative risk reduction compared with warfarin (Odds Ratio [OR] 0.46, 95\% CI $(0.35-0.59) ; p<0.001)$. However, this analysis referred to drug safety only and did not focus on inter-class effects 
regarding prevention of thromboembolic events [55]. Available data for the comparison of severity of vitamin-K-antagonist related $\mathrm{ICH}$ with $\mathrm{NOAC}-\mathrm{ICH}$ provide only little differences, however in-hospital mortality might potentially be reduced in ICH under NOAC $[13,19,38,49]$. So far, studies specifically analyzing NOAC resumption after ICH do currently not exist [25].

\section{Timing of resumption}

The described observational studies documented a median starting point in between 4 to 6 weeks after ICH. Studies more specifically addressing this question reported a broad range of supposed optimal time points ranging from $72 \mathrm{~h}$ to $10-30$ weeks $[15,28]$. One large Swedish registry study $(n=2619)$ suggested an optimal time window within 7-8 weeks for resuming OAC after COX regression-based balancing between observed risk of ischemic and hemorrhagic complications [36]. Although having used sound statistical approaches, limitations of that study comprise censoring the first 4 weeks after $\mathrm{ICH}$, narrow information on patient and $\mathrm{ICH}$ characteristics as well as treatment allocation gathered by outpatient dispensed drug registry [36]. A meta-analysis from the Kings College in London, UK, assessed the associations of resuming VKA six weeks after ICH with occurrence of both thromboembolic and hemorrhagic complications over a one-year follow-up time frame [22]. In essence, VKA-resumption was verified to be safe without increasing hemorrhagic complications over comparator treatments with platelet inhibitors [risk ratio: $1.34,95 \% \mathrm{CI}(0.79-2.30), p=0.28]$, or no antithrombotic treatment respectively [risk ratio: 0.93, 95\% CI (0.45-1.90), $p=0.84$ ] [22]. As yet, it remains unclear when to optimally resume $\mathrm{OAC}$ after $\mathrm{ICH}$ but ongoing randomized trials might provide further evidence. Current expert opinion would suggest a timeframe between 4 to 8 weeks after index ICH depending on patient's individual risk profile [25]. Application of a shorter time period to resumption may only be considered in life-threatening situations and compelling indications, such as symptomatic intracardiac thrombus formation or acute pulmonary embolism, and only after confirmation of hematoma stability by control imaging and strict blood pressure control.

\section{Resumption in patients with mechanical heart valves}

As mentioned above, NOAC should be preferred today for $\mathrm{OAC}$ resumption in indications like $\mathrm{AF}$ or venous thromboembolism. However, this does not apply for patients with mechanical heart valves (MHV) in situ as NOACs were shown to be inferior to VKA in these patients [11]. Neither cardiologic nor neurologic international guidelines provide specific recommendations how to treat MHV patients after ICH [16, 33, 48, 50]. Compared to AF, patients with $\mathrm{MHV}$ are at risk of increased thromboembolic complications why a recent consensus paper from the European Society of Cardiology Working Group in Thrombosis recommended that systemic anticoagulation using heparins may be safe to start as early as 3 days after $\mathrm{ICH}$ and oral anticoagulation using VKA after 7 days, based on limited data from small observational studies and case series [14].

The best available and most recent data came from a sub-group analysis of the German-wide multicenter RETRACE program which included among 2504 OAC-associated ICH patients 166 patients with MHV in situ [26]. Resumption of therapeutic anticoagulation, using heparins or VKA, was related to a 10-fold increase in incidence of major extra- or intracranial hemorrhagic complications during hospital stay in MHV-patients [rate ratio: 10.3, 95\% CI (3.7-35.7)] [26]. Adjusted COX regression modeling for timing of anticoagulation revealed that OAC resumption may be safe after two weeks regarding bleeding complications, whereas optimal balancing of hemorrhagic with thromboembolic complications resulted in an earliest starting point of one week after $\mathrm{ICH}$, to be considered especially in patients with highest risk for thromboembolism, i.e. concomitant AF, mitral position, or older prosthesis types [26]. Main results of this study are summarized in Fig. 1.

\section{Resumption in patients with lobar ICH}

Patients with $\mathrm{ICH}$ in lobar location compared to hypertensive non-lobar located ICH need special considerations due to strong relation to CAA, characterized by deposition of amyloid- $\beta$, micro-hemorrhages, and vascular fragility resulting in a greater risk for $\mathrm{ICH}$ recurrence $[39,45]$. Genetically CAA shows association with apolipoprotein $\mathrm{E}$ alleles (subtypes epsilon 2 \& 4) likely leading to amyloid deposition triggering recurrent $\mathrm{ICH}[16,41]$. A prediction model for CAA-associated lobar ICH integrating genetic characteristics - APOE $\varepsilon 4$ carrier - and radiological CT parameters - subarachnoid hemorrhage, finger-like ICH projections - showed excellent discrimination [Area under the curve: $0.92,95 \% \mathrm{CI}(0.86-0.98)]$ in a recent prospective study [41]. Using MRI and clinical history CAA can be validly identified using the modified Boston criteria [27]. Observational studies documented recurrence rates for patients classified as having definite or probable sporadic CAA of 8.9 per 100 patient-years [95\% CI (7.1-11)] [52].

MRI-detected presence and amount of cerebral microbleeds (CMB) seen on iron-sensitive sequences (T2* weighted gradient-echo or susceptibility-weighted imaging) are associated with both first-ever $\mathrm{ICH}$ in ischemic stroke patients using OAC [OR 2.68, 95\% CI (1.19-6.01), $p=0.017]$ as well as with recurrence of ICH [> 10 CMB versus none: OR 5.6, 95\% CI (2.1-15), $p=0.001$ ] $[8,9]$. Pathologically, CMB correspond to hemosiderin 


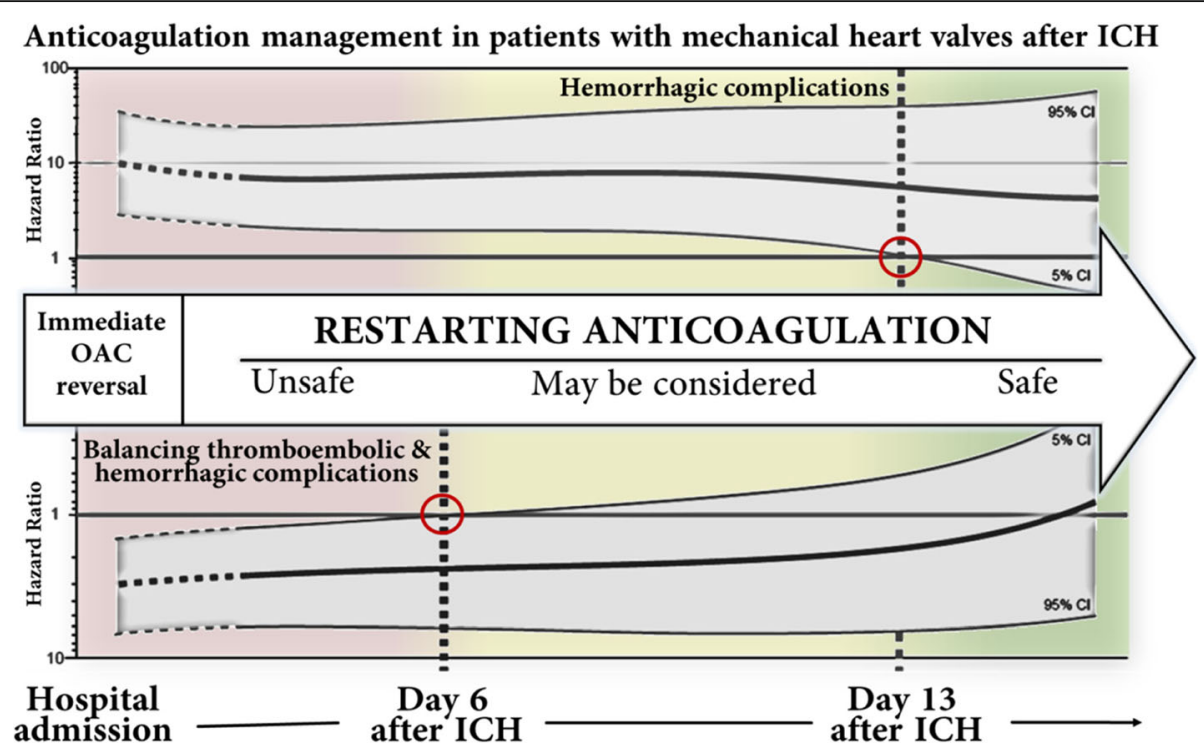

Fig. 1 Suggested in-hospital anticoagulation management in patients with mechanical heart valves according to RETRACE analyses [26]. After initial reversal OAC should not be resumed before day 6 after ICH due to increased hazard for the composite of both thromboembolic and hemorrhagic complications. The hazard for hemorrhagic complications remained significantly increased until day 13. Suggested timeframes should be interpreted with respect to the patient's individual thromboembolic and hemorrhagic risk. Figure modified after Kuramatsu et al., European Heart Journal 2018 [26]. Abbreviations: Cl confidence interval, ICH intracerebral hemorrhage, OAC oral anticoagulation

deposits remaining in macrophages following a self-limiting microhemorrhage [47]. Therefore, they are seen as a neuroimaging marker for small vessel disease contributing to most lobar ICH [54]. Further, cortical superficial siderosis (cSS) and cortical or convexity subarachnoid hemorrhage (cSAH) were identified as independent marker for increased hemorrhagic risk [HR 3.92, 95\% CI (1.3811.17), $p=0.011$, and HR 3.48, 95\% CI (1.13-10.73), $p=0.030$ ] [42]. If both probable CAA and cSS were present one investigation documented an $\mathrm{ICH}$ rate as high as $19 \%$ (95\% CI, 11-32) compared to 6\% (95\% CI, 312 ) in patients without cSS during 5 years of follow-up [7].

Only one sub-analysis of an individual patient data meta-analysis to date aimed to analyze the impact of OAC on $\mathrm{ICH}$ recurrence in patients with probable/possible CAA $(n=190)$. Although OAC resumption was consistent with overall lobar ICH associated with improved functional outcome and decreased mortality, numbers of patients and events were insufficient for investigating the influence of $\mathrm{OAC}$ on complication rates [2]. Summing up, decision making regarding OAC resumption in lobar $\mathrm{ICH}$ patients should include extended diagnostic work-up including magnetic resonance imaging to evaluate characteristics such as a microbleed burden, cSS or cSAH [25]. Their presence should lead to even more critical weighing of the potential benefit of OAC resumption versus the increased bleeding risk, considering also alternative interventional strategies for thromboembolism prevention [25]. For a suggested flow chart on $\mathrm{OAC}$ resumption in patients with deep compared to lobar ICH location please see Fig. 2.

\section{Potential interventional prophylactic alternatives}

The role of the left atrial appendage as the most important source of cardiac thromboembolism related to AF has led to introduction of left atrial appendage occlusion (LAAO) into clinical practice as a potential alternative treatment to long-term OAC. Percutaneous LAAO using the to date only approved device (WATCHMAN) showed non-inferiority compared to warfarin for prophylaxis of a primary composite endpoint of stroke, cardiovascular death, and systemic embolism in the multicenter, randomized PROTECT AF trial including 707 patients with non-valvular atrial fibrillation [18]. As several concerns were raised by the U.S. Food and Drug Administration (FDA) especially regarding early acute safety events, a second trial - PREVAIL - was performed, documenting an improved safety over time (7-day procedure-related complications: PREVAIL 4.2\% versus PROTECT AF $8.7 \%, p=$ $0.004)[17,18]$. However, compared to warfarin this study did only reach non-inferiority regarding stroke or systemic embolism $>7$ days [17]. Regarding the coprimary endpoint of the overall composite of stroke, systemic embolism and cardiovascular/unexplained death (18-month rate ratio 1.07 [0.57-1.89]) the upper bound of 1.89 extended the pre-specified noninferiority margin of 1.75 , triggered by a higher number of (early) strokes in the intervention group (ischemic and hemorrhagic stroke, intervention: 6 [2.2\%] versus control: 1 [0.7\%]) [17]. Next to questionable efficacy and peri-interventional complications, the indicated antithrombotic therapy subsequent to device implantation needs consideration. The recommended antithrombotic 


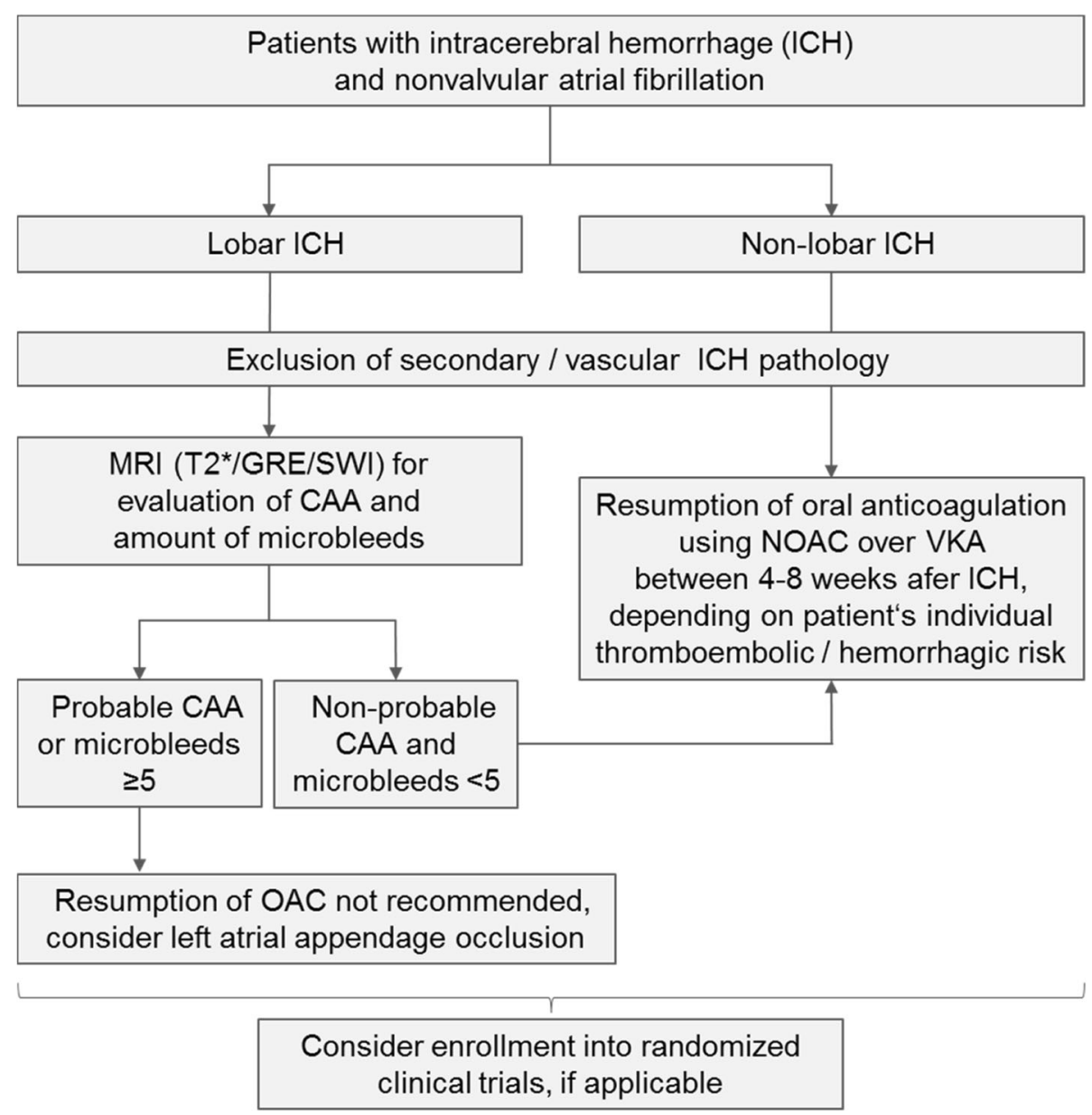

Fig. 2 Suggested flow chart for anticoagulation resumption in ICH patients with nonvalvular atrial fibrillation. Abbreviations: CAA Cerebral amyloid angiopathy, GRE gradient echo, MRI Magnetic resonance imaging, NOAC Non-vitamin K antagonist oral anticoagulants, OAC Oral anticoagulation; Susceptibility-weighted imaging SWI, VKA Vitamin K antagonist

therapy is related to the patient's individual bleeding risk. Based on the PROTECT-AF trial protocol, patients of low bleeding risk should receive VKA for 45 days, and then switch to dual antiplatelet therapy until 6 months, followed by life-long low-dose aspirin monotherapy [18]. Patients of high bleeding risk, as among others patients after $\mathrm{ICH}$, should be treated with dual antiplatelet therapy for one to six months, followed by life-long low-dose aspirin monotherapy. The assumed safety of this approach is based on results from the prospective multicenter nonrandomized ASAP feasibility study investigating LAAO in patients who were ineligible for OAC, however, safety in ICH patients remains unclear [40]. One observational study compared $151 \mathrm{ICH}$ patients with AF who underwent LAAO with a propensity score-matched group of 151 patients receiving standard medical therapy [31]. Analyses showed a decreased risk for the composite endpoint consisting of all-cause mortality, ischemic stroke and major bleeding (HR 0.16, 95\% CI $(0.07-0.37)$ ) as well as for recurrent $\mathrm{ICH}[\mathrm{HR} 0.10,95 \% \mathrm{CI}(0.01-0.81)]$ in patients having LAAO [31]. Therefore, LAAO might potentially represent an alternative strategy to chronic OAC therapy in high-risk ICH patients, provided its successful evaluation in ongoing randomized-controlled trials - especially compared with NOAC as a safer and potentially more effective comparator than VKA $[18,21]$. Today, according to FDA approval, interventional LAAO is formally contraindicated in patients with high-bleeding risk such as ICH patients and its off-label use should be preceded by a critical and interdisciplinary decision making process [43].

\section{Outlook}

Several randomized controlled trials are currently registered in international trial registries investigating both pharmacological treatment and LAAO in patients after intracranial hemorrhage or with high bleeding risk, for overview see Table 1. Pharmacological treatment mostly consist of NOACs (APACHE-AF, ASPIRE, NASPAF-ICH, PRESTIGE-AF) compared to no antithrombotic drug or antiplatelets (all 1:1) whereas interventional trials are 


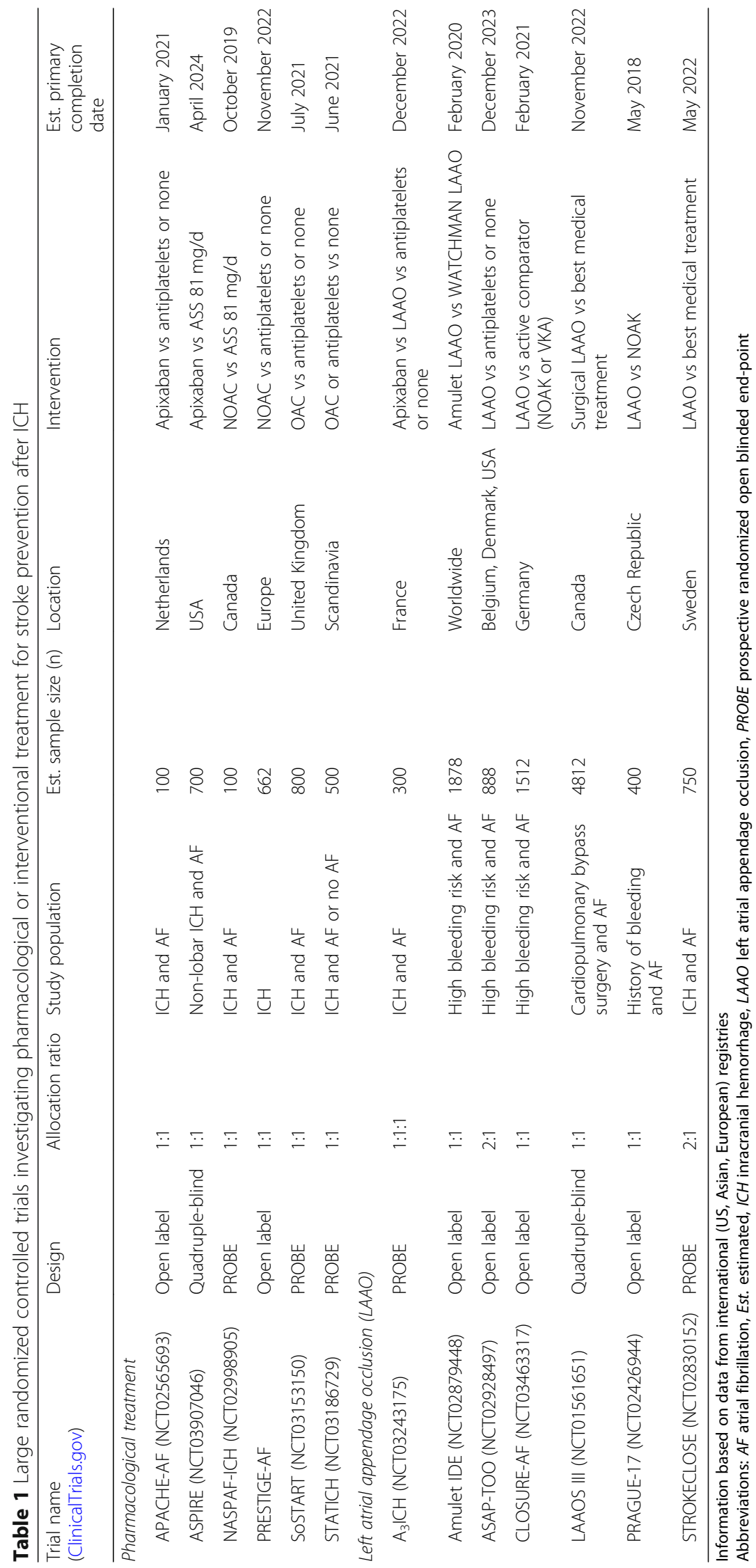


investigating LAAO versus NOAK (PRAGUE-17, 1:1; $\left.\mathrm{A}_{3} \mathrm{ICH}, 1: 1: 1\right)$, or LAOO versus antiplatelets or none (ASAP-TOO, 1:1; $\mathrm{A}_{3} \mathrm{ICH}, 1: 1: 1$ ), or LAAO versus best medical treatment (STROKECLOSE, 2:1; CLOSURE-AF, 1:1; LAAOS III, 1:1), or compare LAOO devices among each other (Amulet IDE, 1:1).

While some trials appear underpowered to detect significant differences (APACHE-AF, NASPAF-ICH, both $n=100$ ), especially three well-designed large pharmacological trials may be able to document statistically significant effects of investigated treatments. First, the investigator-led "Start or STop Anticoagulants Randomised Trial" (SoSTART) plans to include $800 \mathrm{AF}$ patients after non-traumatic and non-aneurysmal intracranial hemorrhage to compare treatment effects of any OAC versus antiplatelets or no antithrombotic medication on the incidence rate of a composite outcome of acute coronary syndrome, ischemic or hemorrhagic stroke, and vascular or non-vascular death. Second, the European Union funded "PREvention of STroke in Intracerebral haemorrhaGE survivors with Atrial Fibrillation" (PRESTIGE-AF) trial aims to include 662 patients with AF after ICH only to investigate superiority of NOACs for prevention of ischemic stroke and non-inferiority regarding recurrence of $\mathrm{ICH}$ compared to antiplatelets or no antithrombotic treatment. Third, the "Anticoagulation in ICH Survivors for PreventIon and REcovery" (ASPIRE) trial plans to include 700 $\mathrm{ICH}$ patients to analyze the hypothesis that Apixaban is superior to ASS for reducing rates of recurrent hemorrhagic or ischemic strokes or death. The strict exclusion of highest-risk patients - lobar ICH with cerebral amyloid angiopathy - prior identified by observational studies may represent a further advantage of this trial.

With respect to LAAO, two trials - STROKECLOSE and $\mathrm{A}_{3} \mathrm{ICH}$ - specifically include patients after $\mathrm{ICH}$ to compare LAAO with pharmacological treatments. Other trials will investigate patients with generally increased bleeding risk, i.e. high HAS-BLED-Scores, history of major bleeding, chronic kidney disease, or per investigator judgement. The German CLOSURE-AF study will analyze the incidence of a composite of stroke, systemic embolism, major bleeding, and cardiovascular or unexplained death, among 1512 patients with $\mathrm{AF}$ and high bleeding risk or history of bleeding (e.g. ICH), comparing LAAO with best medical care including NOAK or VKA. A different approach will be investigated by the quadruple-blind (participant, care provider, investigator, outcomes assessor) trial LAAOS III which will compare surgical LAAO with best medical treatment in patients with AF undergoing surgery for cardiopulmonary bypass.

Taken together, the near future will hopefully provide clear evidence created by large randomized trials to optimally treat patients with indication for oral anticoagulation after $\mathrm{ICH}$.

\section{Conclusions}

Sufficiently powered prospective randomized trials both investigating OAC resumption as well as LAAO are currently recruiting patients. To date, the best evidence comes from large observational studies and meta-analyses, indicating that resumption of $\mathrm{OAC}$ is reducing the risk for thromboembolic events and mortality without significantly increasing the risk for hemorrhagic complications. The use of NOACs might further contribute to safety of OAC resumption, which should take place between 4 to 8 weeks after ICH in patients with AF. Resumption of VKA in patients with MHV should not take place before one week after ICH. Safety may further be influenced by location of $\mathrm{ICH}$ and presence of $\mathrm{CMB}, \mathrm{cSS}$ and $\mathrm{cSAH}$, making it crucial to individually weigh patients' risk for thromboembolic versus hemorrhagic complications.

\section{Abbreviations \\ AF: Atrial fibrillation; CAA: Cerebral amyloid angiopathy; CHADS: [Acronym] Congestive heart failure, Hypertension, Age, Diabetes mellitus, Stroke; $\mathrm{Cl}$ : Confidence interval; CMB: Cerebral microbleeds; $\mathrm{CSAH}$ : cortical or convexity subarachnoid hemorrhage; cSS: cortical superficial siderosis; FDA: Food and Drug Administration; HAS-BLED: [Acronym] Hypertension, Abnormal renal/liver function, Stroke, Bleeding history or predisposition, Labile international normalized ratio, Elderly, Drugs/alcohol concomitantly; HR: Hazard ratio; ICH: Intracerebral hemorrhage; LAAO: Left atrial appendage occlusion; MHV: Mechanical heart valves; NOAC: Non-vitamin K antagonist oral anticoagulants; OAC: Oral anticoagulation; OR: Odds ratio; SUCRA: Surface under the cumulative ranking curve; VKA: Vitamin $\mathrm{K}$ antagonist}

\section{Acknowledgements \\ Not applicable. \\ Funding \\ None. \\ Availability of data and materials \\ Not applicable.}

Authors' contributions

JAS and $\mathrm{HBH}$ prepared the manuscript. JBK and SS critically revised the manuscript for intellectual content. JAS, JBK, HBH performed literature research. All authors read and approved the final manuscript.

Ethics approval and consent to participate Not applicable.

Consent for publication

Not applicable.

Competing interests

The authors declare that they have no competing interests.

\section{Publisher's Note}

Springer Nature remains neutral with regard to jurisdictional claims in published maps and institutional affiliations. 
Received: 10 February 2019 Accepted: 1 April 2019 Published online: 10 May 2019

\section{References}

1. Biffi, A., Anderson, C. D., Battey, T. W., Ayres, A. M., Greenberg, S. M., Viswanathan, A., et al. (2015). Association between blood pressure control and risk of recurrent intracerebral hemorrhage. Jama, 314(9), 904-912.

2. Biffi, A., Kuramatsu, J. B., Leasure, A., Kamel, H., Kourkoulis, C., Schwab, K., et al. (2017). Oral anticoagulation and functional outcome after intracerebral hemorrhage. Annals of Neurology, 82(5), 755-765.

3. Chai-Adisaksopha, C., lorio, A., Hillis, C., Siegal, D., Witt, D. M., Schulman, S., et al. (2017). Warfarin resumption following anticoagulant-associated intracranial hemorrhage: A systematic review and meta-analysis. Thrombosis Research, 160, 97-104.

4. Chao, T. F., Liu, C. J., Liao, J. N., Wang, K. L., Lin, Y. J., Chang, S. L., et al. (2016). Use of Oral anticoagulants for stroke prevention in patients with atrial fibrillation who have a history of intracranial hemorrhage. Circulation, 133(16), 1540-1547.

5. Charidimou, A., Boulouis, G., Gurol, M. E., Ayata, C., Bacskai, B. J., Frosch, M. P. et al. (2017). Emerging concepts in sporadic cerebral amyloid angiopathy. Brain, 140(7), 1829-1850.

6. Charidimou, A., Boulouis, G., Roong piboonsopit, D., Auriel, E., Pasi, M., Haley, K., et al. (2017). Cortical superficial siderosis multifocality in cerebral amyloid angiopathy: A prospective study. Neurology, 89(21), 2128-2135.

7. Charidimou, A., Boulouis, G., Xiong, L., Jessel, M. J., Roongpiboonsopit, D., Ayres, A., et al. (2017). Cortical superficial siderosis and first-ever cerebral hemorrhage in cerebral amyloid angiopathy. Neurology, 88(17), 1607-1614.

8. Charidimou, A., Imaizumi, T., Moulin, S., Biffi, A., Samarasekera, N., Yakushiji, Y. , et al. (2017). Brain hemorrhage recurrence, small vessel disease type, and cerebral microbleeds: A meta-analysis. Neurology, 89(8), 820-829.

9. Charidimou, A., Karayiannis, C., Song, T. J., Orken, D. N., Thijs, V., Lemmens, R., et al. (2017). Brain microbleeds, anticoagulation, and hemorrhage risk: Metaanalysis in stroke patients with AF. Neurology, 89(23), 2317-2326.

10. Chatterjee, S., Sardar, P., Biondi-Zoccai, G., \& Kumbhani, D. J. (2013). New oral anticoagulants and the risk of intracranial hemorrhage: Traditional and Bayesian meta-analysis and mixed treatment comparison of randomized trials of new oral anticoagulants in atrial fibrillation. JAMA Neurology, 70(12), 1486-1490.

11. Eikelboom, J. W., Connolly, S. J., Brueckmann, M., Granger, C. B., Kappetein, A P., Mack, M. J., et al. (2013). Dabigatran versus warfarin in patients with mechanical heart valves. The New England Journal of Medicine, 369(13), 1206-1214.

12. Gage, B. F., van Walraven, C., Pearce, L., Hart, R. G., Koudstaal, P. J., Boode, B. S., et al. (2004). Selecting patients with atrial fibrillation for anticoagulation: Stroke risk stratification in patients taking aspirin. Circulation, 110(16), 2287-2292.

13. Gerner, S. T., Kuramatsu, J. B., Sembill, J. A., Sprugel, M. I., Endres, M. Haeusler, K. G., et al. (2018). Association of prothrombin complex concentrate administration and hematoma enlargement in non-vitamin $\mathrm{K}$ antagonist oral anticoagulant-related intracerebral hemorrhage. Annals of Neurology, 83(1), 186-196.

14. Halvorsen, S., Storey, R. F., Rocca, B., Sibbing, D., Ten Berg, J., Grove, E. L., et al. (2017). Management of antithrombotic therapy after bleeding in patients with coronary artery disease and/or atrial fibrillation: Expert consensus paper of the European Society of Cardiology Working Group on thrombosis. European Heart Journal, 38(19), 1455-1462.

15. Hawryluk, G. W., Austin, J. W., Furlan, J. C., Lee, J. B., O'Kelly, C., \& Fehlings, M. G. (2010). Management of anticoagulation following central nervous system hemorrhage in patients with high thromboembolic risk. Journal of Thrombosis and Haemostasis, 8(7), 1500-1508.

16. Hemphill, J. C., 3rd, Greenberg, S. M., Anderson, C. S., Becker, K., Bendok, B. R., Cushman, M., et al. (2015). Guidelines for the Management of Spontaneous Intracerebral Hemorrhage: A guideline for healthcare professionals from the American Heart Association/American Stroke Association. Stroke, 46(7), 2032-2060.

17. Holmes, D. R., Jr., Kar, S., Price, M. J., Whisenant, B., Sievert, H., Doshi, S. K., et al. (2014). Prospective randomized evaluation of the Watchman left atrial appendage closure device in patients with atrial fibrillation versus long-term warfarin therapy: The PREVAIL trial. Journal of the American College of Cardiology, 64(1), 1-12.

18. Holmes, D. R., Reddy, V. Y. Turi, Z. G., Doshi, S. K., Sievert, H., Buchbinder, M. et al. (2009). Percutaneous closure of the left atrial appendage versus warfarin therapy for prevention of stroke in patients with atrial fibrillation: A randomised non-inferiority trial. Lancet, 374(9689), 534-542.

19. Inohara, T., Xian, Y., Liang, L., Matsouaka, R. A., Saver, J. L., Smith, E. E., et al. (2018). Association of Intracerebral Hemorrhage among Patients Taking non-Vitamin K Antagonist vs vitamin $\mathrm{K}$ antagonist Oral anticoagulants with in-hospital mortality. JAMA, 319(5), 463-473.

20. Katsanos, A. H., Schellinger, P. D., Kohrmann, M., Filippatou, A., Gurol, M. E., Caso, V., et al. (2018). Fatal oral anticoagulant-related intracranial hemorrhage: A systematic review and meta-analysis. European Journal of Neurology, 25(10), 1299-1302.

21. Kirchhof, P., Benussi, S., Kotecha, D., Ahlsson, A., Atar, D., Casadei, B., et al. (2016). 2016 ESC guidelines for the management of atrial fibrillation developed in collaboration with EACTS. European Heart Journal, 37(38), 2893-2962.

22. Korompoki, E., Filippidis, F. T., Nielsen, P. B., Del Giudice, A., Lip, G. Y. H., Kuramatsu, J. B., et al. (2017). Long-term antithrombotic treatment in intracranial hemorrhage survivors with atrial fibrillation. Neurology, 89(7), 687-696.

23. Krishnamurthi, R. V., Feigin, V. L., Forouzanfar, M. H., Mensah, G. A., Connor, M., Bennett, D. A., et al. (2013). Global and regional burden of first-ever ischaemic and haemorrhagic stroke during 1990-2010: Findings from the global burden of disease study 2010. The Lancet Global Health, 1(5), e259-e281.

24. Kuramatsu, J. B., Gerner, S. T., Schellinger, P. D., Glahn, J., Endres, M., Sobesky, J., et al. (2015). Anticoagulant reversal, blood pressure levels, and anticoagulant resumption in patients with anticoagulation-related intracerebral hemorrhage. JAMA, 313(8), 824-836.

25. Kuramatsu, J. B., \& Huttner, H. B. (2019). Management of oral anticoagulation after intracerebral hemorrhage. International Journal of Stroke, 14(3), 238-246.

26. Kuramatsu, J. B., Sembill, J. A., Gerner, S. T., Sprugel, M. I., Hagen, M., Roeder, S. S., et al. (2018). Management of therapeutic anticoagulation in patients with intracerebral haemorrhage and mechanical heart valves. European Heart Journal, 39(19), 1709-1723.

27. Linn, J., Halpin, A., Demaerel, P., Ruhland, J., Giese, A. D., Dichgans, M., et al. (2010). Prevalence of superficial siderosis in patients with cerebral amyloid angiopathy. Neurology, 74(17), 1346-1350.

28. Majeed, A., Kim, Y. K., Roberts, R. S., Holmstrom, M., \& Schulman, S. (2010). Optimal timing of resumption of warfarin after intracranial hemorrhage. Stroke, 41(12), 2860-2866.

29. Murphy, M. P., Kuramatsu, J. B., Leasure, A., Falcone, G. J., Kamel, H., Sansing, L. H., et al. (2018). Cardioembolic stroke risk and recovery after anticoagulation-related intracerebral hemorrhage. Stroke, 49(11), 2652-2658.

30. Murthy, S. B., Gupta, A., Merkler, A. E., Navi, B. B., Mandava, P., ladecola, C., et al. (2017). Restarting anticoagulant therapy after intracranial hemorrhage: A systematic review and meta-analysis. Stroke, 48(6), 1594-1600.

31. Nielsen-Kudsk, J. E., Johnsen, S. P., Wester, P., Damgaard, D., Airaksinen, J., Lund, J., et al. (2017). Left atrial appendage occlusion versus standard medical care in patients with atrial fibrillation and intracerebral haemorrhage: A propensity score-matched follow-up study. Eurolntervention, 13(3), 371-378.

32. Nielsen, P. B., Larsen, T. B., Skjoth, F., Gorst-Rasmussen, A., Rasmussen, L. H., \& Lip, G. Y. (2015). Restarting anticoagulant treatment after intracranial hemorrhage in patients with atrial fibrillation and the impact on recurrent stroke, mortality, and bleeding: A Nationwide cohort study. Circulation, 132(6), 517-525.

33. Nishimura, R. A., Otto, C. M., Bonow, R. O., Carabello, B. A., Erwin, J. P., 3rd, Fleisher, L. A., et al. (2017). 2017 AHA/ACC focused update of the 2014 AHA ACC guideline for the Management of Patients with Valvular Heart Disease: A report of the American College of Cardiology/American Heart Association task force on clinical practice guidelines. Circulation, 135(25), e1159-e1195.

34. Ottosen, T. P., Grijota, M., Hansen, M. L., Brandes, A., Damgaard, D., Husted, S. E., et al. (2016). Use of antithrombotic therapy and long-term clinical outcome among patients surviving intracerebral hemorrhage. Stroke, 47(7), 1837-1843.

35. Park, Y. A., Uhm, J. S., Pak, H. N., Lee, M. H., \& Joung, B. (2016). Anticoagulation therapy in atrial fibrillation after intracranial hemorrhage. Heart Rhythm, 13(9), 1794-1802.

36. Pennlert, J., Overholser, R., Asplund, K., Carlberg, B., Van Rompaye, B., Wiklund, P. G., et al. (2017). Optimal timing of anticoagulant treatment after intracerebral hemorrhage in patients with atrial fibrillation. Stroke, 48(2), 314-320.

37. Pisters, R., Lane, D. A., Nieuwlaat, R., de Vos, C. B., Crijns, H. J., \& Lip, G. Y. (2010). A novel user-friendly score (HAS-BLED) to assess 1-year risk of major bleeding in patients with atrial fibrillation: The euro heart survey. Chest, 138(5), 1093-1100. 
38. Purrucker, J. C., Haas, K., Rizos, T., Khan, S., Wolf, M., Hennerici, M. G., et al. (2016). Early clinical and radiological course, management, and outcome of intracerebral hemorrhage related to new Oral anticoagulants. JAMA Neurology, 73(2), 169-177.

39. Qureshi, A. I., Mendelow, A. D., \& Hanley, D. F. (2009). Intracerebral haemorrhage. Lancet, 373(9675), 1632-1644.

40. Reddy, V. Y., Mobius-Winkler, S., Miller, M. A., Neuzil, P., Schuler, G., Wiebe, J., et al. (2013). Left atrial appendage closure with the Watchman device in patients with a contraindication for oral anticoagulation: The ASAP study (ASA Plavix feasibility study with Watchman left atrial appendage closure technology). Journal of the American College of Cardiology, 61(25), 2551-2556.

41. Rodrigues, M. A., Samarasekera, N., Lerpiniere, C., Humphreys, C., McCarron, M. O., White, P. M., et al. (2018). The Edinburgh CT and genetic diagnostic criteria for lobar intracerebral haemorrhage associated with cerebral amyloid angiopathy: Model development and diagnostic test accuracy study. Lancet Neurology, 17(3), 232-240.

42. Roongpiboonsopit, D., Charidimou, A., William, C. M., Lauer, A., Falcone, G. J., Martinez-Ramirez, S., et al. (2016). Cortical superficial siderosis predicts early recurrent lobar hemorrhage. Neurology, 87(18), 1863-1870.

43. Schellinger, P. D., Tsivgoulis, G., Steiner, T., \& Kohrmann, M. (2018). Percutaneous left atrial appendage occlusion for the prevention of stroke in patients with atrial fibrillation: Review and critical appraisal. J Stroke, 20(3), 281-291.

44. Sembill, J. A., Gerner, S. T., Volbers, B., Bobinger, T., Lucking, H., Kloska, S. P., et al. (2017). Severity assessment in maximally treated ICH patients: The max-ICH score. Neurology, 89(5), 423-431.

45. Sembill, J. A., Huttner, H. B., \& Kuramatsu, J. B. (2018). Impact of recent studies for the treatment of intracerebral hemorrhage. Current Neurology and Neuroscience Reports, 18(10), 71.

46. Sembill, J. A., Wieser, C. Y., Sprugel, M. I., Gerner, S. T., Giede-Jeppe, A., Reindl, C., et al. (2018). Initiating anticoagulant therapy after $\mathrm{ICH}$ is associated with patient characteristics and treatment recommendations. Journal of Neurology, 265(10), 2404-2414.

47. Shoamanesh, A., Kwok, C. S., \& Benavente, O. (2011). Cerebral microbleeds: Histopathological correlation of neuroimaging. Cerebrovascular Diseases, 32(6), 528-534.

48. Steiner, T., Al-Shahi Salman, R., Beer, R., Christensen, H., Cordonnier, C., Csiba, L., et al. (2014). European stroke organisation (ESO) guidelines for the management of spontaneous intracerebral hemorrhage. International Journal of Stroke.

49. Tsivgoulis, G., Lioutas, V. A., Varelas, P., Katsanos, A. H., Goyal, N., Mikulik, R. et al. (2017). Direct oral anticoagulant- vs vitamin K antagonist-related nontraumatic intracerebral hemorrhage. Neurology, 89(11), 1142-1151.

50. Vahanian, A., Alfieri, O., Andreotti, F., Antunes, M. J., Baron-Esquivias, G., Baumgartner, H., et al. (2012). Guidelines on the management of valvular heart disease (version 2012). European Heart Journal, 33(19), 2451-2496.

51. van Asch, C. J., Luitse, M. J., Rinkel, G. J., van der Tweel, I., Algra, A., \& Klijn, C. J. (2010). Incidence, case fatality, and functional outcome of intracerebral haemorrhage over time, according to age, sex, and ethnic origin: A systematic review and meta-analysis. Lancet Neurology, 9(2), 167-176.

52. van Etten, E. S., Gurol, M. E., van der Grond, J., Haan, J., Viswanathan, A., Schwab, K. M., et al. (2016). Recurrent hemorrhage risk and mortality in hereditary and sporadic cerebral amyloid angiopathy. Neurology, 87(14), 1482-1487.

53. Vestergaard, A. S., Skjoth, F., Lip, G. Y., \& Larsen, T. B. (2016). Effect of anticoagulation on hospitalization costs after intracranial hemorrhage in atrial fibrillation: A registry study. Stroke, 47(4), 979-985.

54. Wardlaw, J. M., Smith, E. E., Biessels, G. J., Cordonnier, C., Fazekas, F., Frayne, R., et al. (2013). Neuroimaging standards for research into small vessel disease and its contribution to ageing and neurodegeneration. Lancet Neurology, 12(8), 822-838.

55. Wolfe, Z., Khan, S. U., Nasir, F., Raghu Subramanian, C., \& Lash, B. (2018). A systematic review and Bayesian network meta-analysis of risk of intracranial hemorrhage with direct oral anticoagulants. Journal of Thrombosis and Haemostasis, 16(7), 1296-1306.

56. Yung, D., Kapral, M. K., Asllani, E., Fang, J., \& Lee, D. S. (2012). Reinitiation of anticoagulation after warfarin-associated intracranial hemorrhage and mortality risk: The best practice for reinitiating anticoagulation therapy after intracranial bleeding (BRAIN) study. The Canadian Journal of Cardiology, 28(1), 33-39.

57. Zhou, Z., Yu, J., Carcel, C., Delcourt, C., Shan, J., Lindley, R. I., et al. (2018). Resuming anticoagulants after anticoagulation-associated intracranial haemorrhage: Systematic review and meta-analysis. BMJ Open, 8(5), e019672.

\section{Ready to submit your research? Choose BMC and benefit from:}

- fast, convenient online submission

- thorough peer review by experienced researchers in your field

- rapid publication on acceptance

- support for research data, including large and complex data types

- gold Open Access which fosters wider collaboration and increased citations

- maximum visibility for your research: over $100 \mathrm{M}$ website views per year

At BMC, research is always in progress.

Learn more biomedcentral.com/submissions 